Results The average age of patients was 46 years, with men accounted for $69 \%$ of the total. After treatment, normalization of ALT $71.26 \%$, the viral response of $90.23 \%$, HBV DNA below the detection level was $66.3 \%$. Liver fibrosis evaluated by FibroScan before and after 6 months treatment were 7.15 $\pm 1.56 \mathrm{kPa}$, and $3.58 \pm 1.19 \mathrm{kPa}$ evaluated by FibroScan. Conclusions TDF was effective for patients after treatment on liver fibrosis assessed by FibroScan in chronic hepatitis B patients.

\section{IDDF2020-ABS-0199 HISTOPATHOLOGICAL AND IMMUNOHISTOCHEMICAL CHARACTERIS- TICS OF HEPATOCELLULAR CARCINOMA WITH PORTAL VEIN THROMBOSIS}

${ }^{1}$ Le Thi Thu Hien*, ${ }^{2}$ Le Quoc Tuan, ${ }^{3}$ Nguyen Quang Duat. 'Department of Internal Medicine, Thai Nguyen University of Medicine and Pharmacy, Vietnam; ${ }^{2}$ Department of Internal Medicine, Thanh Ba Hospital, Vietnam; ${ }^{3}$ Department of Internal Medicine, 103 Military Hospital, Vietnam

\subsection{6/gutjpl-2020-IDDF.168}

Background Detection of portal vein thrombosis (PVT), especially benign or neoplastic PVT in new diagnosed hepatocellular carcinoma (HCC) has key meaning for prognosis as well as making the choice of treatment methods.

Objectives To analysis of histopathological and immunohistochemical characteristics of hepatocellular carcinoma with portal vein thrombosis

(1)To the analysis of histopathological and immunohistochemical characteristics of hepatocellular carcinoma with portal vein thrombosis

(2)To analyse the histopathological and immunohistochemical characteristics of hepatocellular carcinoma with portal vein thrombosis

Methods We conducted a prospective study of 50 patients with HCC have PVT at Clinic 103 Cam Khe from June 2017 to August 2019. The PVT specimens were collected by biopsy through the skin according to ultrasound guidance. Immunohistochemistry test: the dyeing was performed by BondMax automatic dyeing machine (Leica Biosystems - Australia).

Results The location of HCC tumors associated with the location of PVT $(p<0.01)$. Thromboses in the trunk of the portal vein of $56 \%$, thromboses in the branches of the portal vein of $44 \%$. The degree of thrombosis significantly related to the size of HCC tumor $(\mathrm{p}<0.01)$. All of the PVT in patients with HCC were malignant thromboses with moderate differentiation of $58 \%$, poor differentiation of $36 \%$, high differentiation of $6 \%$. Neoangiogenesis in the thromboses by using immunohistochemistry: low level of 6\%, moderate level of $46 \%$ and a high level of $48 \%$. The neoangiogenesis in the thromboses significantly related to cancer cell differentiation $(\mathrm{p}<0.01)$.

Conclusions The more angiogenesis was, the lower the grate of cell differentiation.

\section{IDDF2020-ABS-0210 STATIN USE ASSOCIATED WITH REDUCED RISK OF ALL-CAUSE MORTALITY IN HEPATOCELLULAR CARCINOMA PATIENTS FOLLOWING LIVER RESECTION: A SYSTEMATIC REVIEW AND META- ANALYSIS}

Philip Chun Yeung*, Siu Tim Cheung, Kelvin Kwok-Chai Ng, Paul Bo-San Lai, Charing Ching-Ning Chong. The Chinese University of Hong Kong, Hong Kong

\subsection{6/gutjnl-2020-IDDF.169}

Background The benefit of statins in overall survival of hepatocellular carcinoma (HCC) patients after curative liver resection has been controversial. Some retrospective studies identified perioperative use of statins was associated with significantly reduction in all-cause mortality in HCC patients after liver resection, among patients with or without chronic hepatitis viral infection in multivariate survival analysis. However, there are also several studies conducted in Japan and United States against the claim. To evaluate the effect of statins on the risk of all-cause mortality in HCC patients after curative liver resections, we performed a systematic review and meta-analysis on this topic.

Methods A systematic search of Medline, Embase, Cochrane Library and Web of Science was conducted through August 2020. Studies were included if they evaluated perioperative exposure to statins, reported the all-cause mortality of HCC patients after curative resection and reported adjusted hazard ratios (HR) of multivariable analysis by Cox proportional hazards model. Summary HR estimates with 95\% confidence intervals (CI) were calculated using the random-effects model.

Results The analysis included 4 studies reporting the all-cause mortality of 3,762 HCC patients after liver resection, in which 384 of them received perioperative statins treatments. A meta-analysis of the studies showed a significant (48\%) reduction in the risk of all-cause mortality among HCC patients who had perioperative statins use (adjusted HR, 0.52; 95\% CI, 0.34-0.82), with moderate heterogeneity among studies (Cochran's $\mathrm{Q}$ test, $\mathrm{P}=0.12, \mathrm{I}^{2}=49 \%$ ) (figure 1) with no publication bias. patients after liver resection

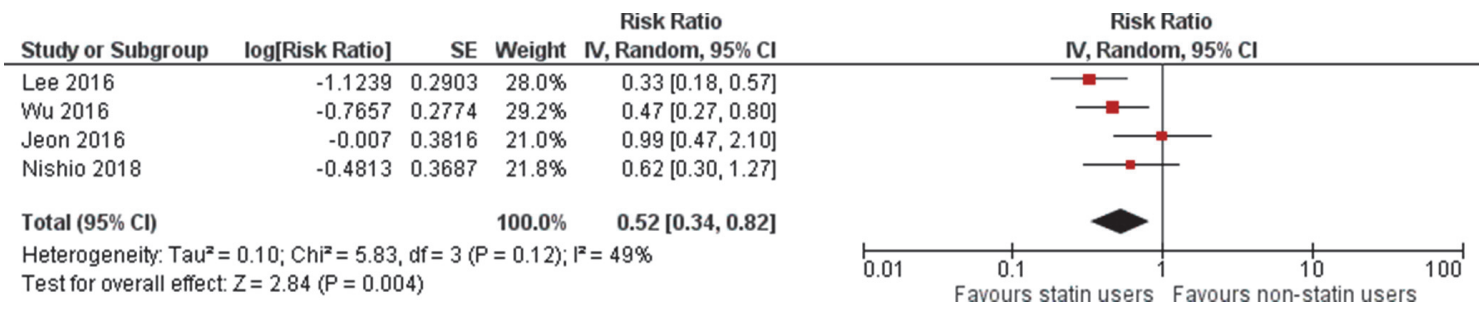

Abstract IDDF2020-ABS-0210 Figure 1 Forest plot for the meta-analysis of the association between statin use and overall survival in HCC 
Conclusions In conclusion, perioperative statins use may reduce the risk of all-cause mortality of HCC patients after curative liver resection. Further prospective studies and larger randomized controlled trials (RCTs) are warranted to validate the conclusion.

\section{IDDF2020-ABS-0218 THE VALUES OF ACOUSTIC RADIATION FORCE IMPULSE ELASTOGRAPHY OF LIVER IN EVALUATING THE DEGREE OF LIVER FIBROSIS IN ALCOHOLIC LIVER DISEASE}

${ }^{1}$ Ha Phuong Linh*, ${ }^{2}$ Tran Huu Ngoc. 'Department of Internal Medicine, 103 Cam Khe Clinic, Vietnam; ' 2 Department of Internal Medicine, Hung Vuong Hospital, Vietnam

\subsection{6/gutjnl-2020-IDDF.170}

Background Prevalence of alcoholic liver disease (ALD) is increasingly elevated all over the world. The degree of liver fibrosis (LF) is an important factor that affects the treatment and prognosis. Acoustic radiation force impulse (ARFI) elastography is a noninvasive and a new way of assessing LF.

Aims To evaluate the diagnostic performance of ARFI elastography for assessing LF in ALD.

Methods This prospective study included 40 patients with ALD at Thai Nguyen National Hospital from June 2019 to February 2020. These patients had been diagnosed with ALD according to the guideline of the AASL 2010. Collecting samples for laboratory testing. Liver stiffness measurement was done using ARFI elastography. Liver biopsy was graded using Metavir classification.

Results Majority were in age group of 41-50 years (63\%). The mean ARFI elastometry showed $2.71 \pm 0.59 \mathrm{~m} / \mathrm{s}$ (range $0.86-4.53$ ) in 40 male patients. Shear wave velocity (SWV) significantly correlated with the fibrosis stage. The area under the ROC curves (AUROCs) for diagnosis of $\geq F 2$ and $\geq$ F3 were 0.87 (95\% CI: $0.78-0.95)$ and 0.89 (95\% CI: $0.81-0.97)$, respectively. The cut-off values of SWV for $\geq$ F2 and $\geq$ F3 were $1.98 \mathrm{~m} / \mathrm{s}$ (Se 78.25\%, Sp 88.37\%, PPV $85.6 \%$ and NPV $82.4 \%$ ) and $\geq 2.31 \mathrm{~m} / \mathrm{s}$ (Se 95.68\%, Sp 85.58\%, PPV 71.6\%, NPV 97.8\%), respectively. APRI significantly correlated with the fibrosis stage. AUROCs for diagnosis of $\geq$ F2 and $\geq$ F3 were 0.8 (95\% CI: $0.58-0.76)$ and 0.78 (95\% CI: $0.84-0.82$ ), respectively. The cut-off values of APRI for diagnosis of $\geq$ F2 and $\geq$ F3 were $\geq 0.62$ (Se $51.1 \%$, Sp $87.13 \%$, PPV $72.3 \%$ and NPV 68.1\%) and $\geq$ 1.171 (Se 42\%, Sp 95.52\%, PPV 81\%, NPV 83.1\%), respectively.

Conclusions Increasing SWV correlates with a higher degree of LF. ARFI elastography is a noninvasive, reliable, and repeatable diagnostic test in evaluating the degree of LF.

\section{IDDF2020-ABS-0219 NEUTROPHIL LYMPHOCYTE RATIO AND PLATELET LYMPHOCYTE RATIO ARE EXCELLENT MARKERS FOR PREDICTING SURVIVAL AND SEVERITY OF HEPATOCELLULAR CARCINOMA}

Sagar Walinjkar*, Anil Arora, Ashish Kumar, Praveen Sharma, Naresh Bansal. Institute of Liver, Gastroenterology and Pancreaticobiliary Sciences, Sir Ganga Ram Hospital, India
Background Hepatocellular carcinoma (HCC) is the 5th most common malignancy worldwide. Its clinical presentation in terms of its severity is quite variable, and survival in its advanced stages is poor. Neutrophil lymphocyte ratio (NLR) and Platelet lymphocyte ratio (PLR) are the markers which have been proven useful in prognostication of many malignancies. We aimed to evaluate the predictive value of NLR and PLR in prognosticating patients with HCC and to study its association with severity of HCC.

Methods Patients with newly diagnosed HCC getting admitted between Aug 2018 and Jan 2020 were included in the study. The imaging features, ECOG status and liver functional status were analyzed, and BCLC staging was done. Patients were given appropriate treatment according to the BCLC stage. Pretreatment NLR and PLR were calculated from differential leukocyte count. Repeat imaging was done at 1 and 3 months post-treatment. Patients were followed up for 6 months for survival. Optimum cut off values of NLR and PLR were calculated by ROC curve analysis. The OS at 6 months was compared on Kaplan Meier curve using high and low NLR, PLR values. Association between NLR and PLR was also tested with BCLC stages, recurrence-free and progression-free survival.

\section{OVERALL SURVIVAL AT 6 MONTHS}
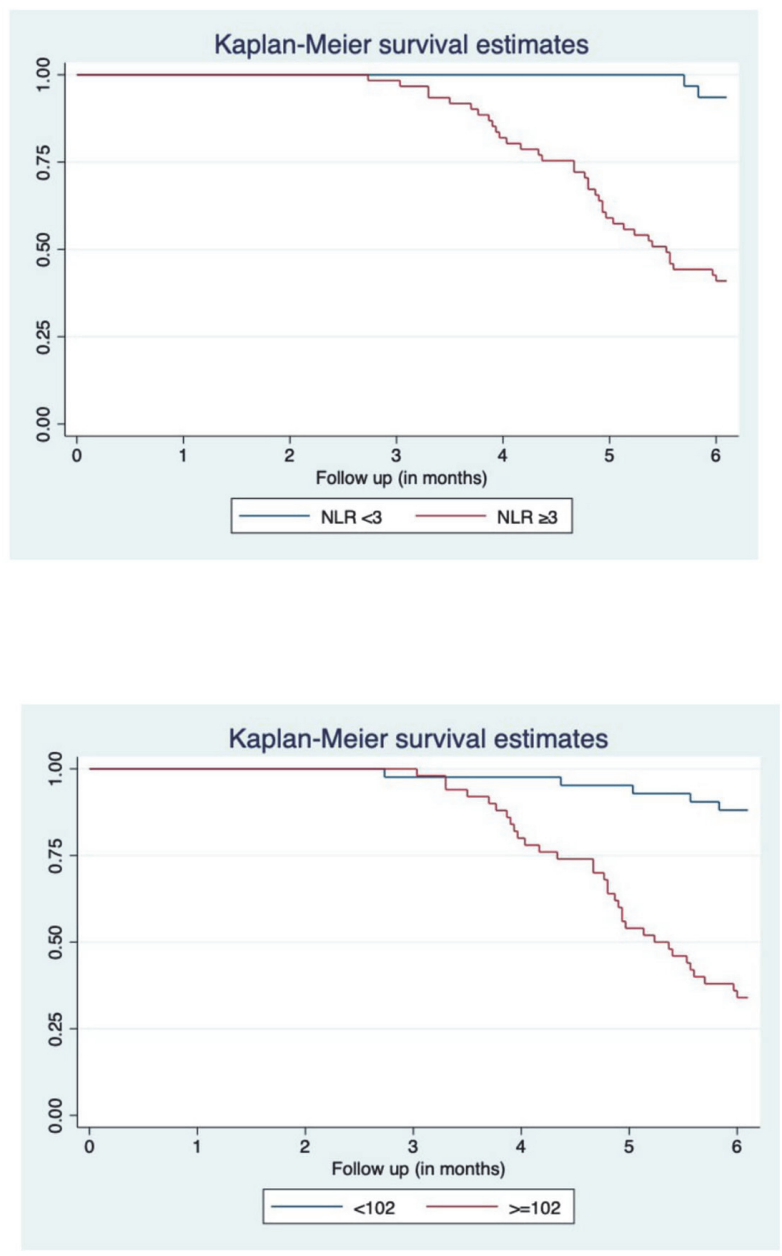

Abstract IDDF2020-ABS-0219 Figure 1 Kaplan meier curves for overall survival at 6 months according to NLR and PLR 